\title{
Sirolimus and MMF are insufficient immunosuppressants for regulation of the proliferation of CD133+EpCAM+ cell populations in $\mathrm{HCC}$ cell lines
}

\author{
HWAJUNG KIM, KWANG-WOONG LEE, SEUNG CHEOL OH, MIN-YOUNG PARK, SOOIN SEO, \\ XUE-LI JIN, SUK KYUN HONG, KYUNG CHUL YOON, NAM-JOON YI and KYUNG-SUK SUH \\ Department of Surgery, Seoul National University College of Medicine, Seoul 03080, South Korea
}

Received January 9, 2020; Accepted July 22, 2020

DOI: $10.3892 /$ br.2020.1376

\begin{abstract}
Studies on effective immunosuppressive strategies for the management of patients undergoing a liver transplantation (LT) due to hepatocellular carcinoma (HCC) are limited. In the present study, immunosuppressive candidates predicted to exhibit beneficial immunosuppressive and tumor-suppressive effects in patients with HCC were assessed using Huh7 and HEP3B HCC cells, which have high proportions of CD133+EpCAM+ cancer stem cell (CSC) populations. The immunosuppressants assessed were sirolimus, tacrolimus, cyclosporine A and mycophenolate mofetil (MMF), and their activities were assessed on CSCs. Sirolimus and MMF reduced the proliferation of Huh7 and HEP3B cells; however, the proportion of CD133+EpCAM+ was notably increased in treated Huh7 cells. Sirolimus treatment alone resulted in G0-G1 cell cycle arrest at all doses in all Huh7 and CD133-EpCAM- populations; however, CD133+EpCAM+ populations showed only slight G1 arrest at higher doses only. In contrast, S-phase arrest was induced at all doses in the Huh7, CD133-EpCAM- and CD133+EpCAM+ populations by MMF. Sirolimus and MMF effectively reduced the proliferation of Huh7 and HEP3B cells, but did not exert a notable effect on the CD133+EpCAM+ cells. Therefore, therapeutic strategies utilizing Sirolimus and MMF should be further studied in vivo
\end{abstract}

Correspondence to: Professor Kwang-Woong Lee, Department of Surgery, Seoul National University College of Medicine, 101 Daehang-ro, Jongro-gu, Seoul 03080, South Korea

E-mail:kwleegs@gmail.com

Abbreviations: CNI, calcineurin inhibitors; CSC, cancer stem cell; EpCAM, epithelial cell adhesion molecule; HCC, hepatocellular carcinoma; IHC, immunohistochemistry; LT, liver transplantation; MMF, mycophenolate mofetil; mTORI, mammalian target of rapamycin inhibitor

Key words: liver transplantation, CSC, immunosuppressants, hepatocellular carcinoma, CD133, EpCAM for regulation of CSC populations in order to reduce HCC recurrence rates.

\section{Introduction}

Hepatocellular carcinoma (HCC) is the fifth most common type of cancer and the third leading cause of cancer-associated death worldwide $(1,2)$. Liver transplantation (LT) is becoming an increasingly used treatment strategy for patients with early stage unresectable HCC, as it offers complete tumor excision along with the removal of the carcinogenic liver. However, the incidence of tumor recurrence following LT is reported to be between $15-24 \%$ and it is the primary cause of death for patients with HCC who have undergone LT (3-5). To prevent rejection of the liver graft, patients require life-long immunosuppression using appropriate drugs (3-5). Unfortunately, immunosuppressants exhibit a range of side-effects, with some immunosuppressive drugs possessing tumorigenic capacity (6). It is therefore important to develop drugs that demonstrate both immunosuppressive and tumor-suppressive effects to effectively treat patients, prolonging their survival and reducing the potential for tumor recurrence.

The choice of immunosuppressive regimen is paramount, as several studies have shown that tumor progression is more rapid and aggressive in immunosuppressed patients following LT, and the degree of immunosuppression negatively affects post-LT recurrence of HCC, and thus, long-term survival of these patients (6). Traditionally, the most commonly used immunosuppressive regimen following LT is a calcineurin inhibitor (CNI) based regimen. CNIs, such as tacrolimus and cyclosporine $\mathrm{A}$, have been demonstrated to possess pro-oncogenic effects both in experimental models, and in retrospective and prospective clinical trials (7). Mycophenolate mofetil (MMF) possesses anti-proliferative properties, but has not been shown to prevent HCC recurrence $(7,8)$. However, mammalian target of rapamycin inhibitors (mTORIs), such as sirolimus and everolimus, possess anti-tumor properties (9) and are of particular interest for use in LT patients with HCC. Studies have shown promising results for both sirolimus and everolimus, highlighting their beneficial effects on post transplantation recurrence of HCC (10-12). 
A high risk of recurrence may be associated with any remaining cancer stem cells (CSCs) following therapy as well as immunosuppression after LT (13-15). The CSC theory provides novel insights into the formation of tumors. Tumors are organized into a hierarchy of heterogeneous cell populations, a small subset of which are termed CSCs or tumor-initiating cells, and these cells exhibit the ability to drive and sustain tumor growth (16). Cancer stem/progenitor cells have been identified in several studies in patients with HCC (13-15). Therefore, it is imperative to target and control the remaining or circulating CSCs following LT to prevent recurrence. As such the suitability of immunosuppression for targeting CSCs for prevention of recurrence in LT patients should be considered.

In the present study, the role of potentially suitable immunosuppressive candidates predicted to exhibit beneficial immunosuppressive and tumor-suppressive effects in patients with $\mathrm{HCC}$ were assessed utilizing a number of common HCC cell lines and their CSC populations. The specific expression levels of the CSC markers CD133, epithelial cell adhesion molecule (EpCAM) and CD90 in multiple HCC cell lines was determined. Additionally, the inhibition of proliferation on each of these cell lines with the most commonly used LT immunosuppressant drugs (sirolimus, tacrolimus, cyclosporin A and MMF) was evaluated. The aim of the present study was to provide a clinically relevant treatment model with optimal immunosuppressive effects for the best potential outcome in patients undergoing LT for HCC.

\section{Materials and methods}

Cell lines and culture. Human HCC cell lines Huh7, Hep3B, SNU182, SNU387 and SNU449 used in the present study were obtained from the American Type Culture Collection. Huh7, Hep3B, SNU182, SNU387 and SNU449 cells were cultured in DMEM supplemented with 10\% FBS (Gibco; Thermo Fisher Scientific, Inc.), $1 \mathrm{mM}$ sodium pyruvate, $100 \mathrm{U}$ penicillin and $100 \mathrm{mg}$ streptomycin (Sigma-Aldrich; Merck KGaA) at $37^{\circ} \mathrm{C}$ in a humidified incubator with $5 \% \mathrm{CO}_{2}$.

Staining with stem cell markers using fluorescence activated cell sorting (FACS). As CSC markers, anti-CD133, anti-epithelial cell adhesion molecule (EpCAM), anti-CD44 and anti-CD90 were used in the present study. The expression levels of these CSC markers in each of the four HCC cell lines were analyzed using FACS. Cells were dissociated and re-suspended in PBS containing 0.5\% BSA. Flow cytometry was performed using phycoerythrin (PE)-conjugated anti-human CD133 antibody (1:100; cat. no. 130-113-670; Miltenyi Biotec) and fluorescein isothiocyanate (FITC) conjugated anti-human EpCAM antibody (1:100; cat. no. ab8666; Abcam), PE-conjugated CD44 (1:50; cat. no. 130-113-336; Miltenyi Biotec) and FITC-conjugated CD45 (1:20 cat. no. 103107; BioLegend, Inc.) or PE-conjugated CD90 (1:50; cat. no. 555596; BD Biosciences). Staining patterns were visualized using flow cytometry [FACS Canto II (HTS); BD Biosciences]. Data analysis was performed using FACSDiva software (BD Biosciences).
Immunosuppressive agents. Sirolimus was obtained from Pfizer, Inc. and tacrolimus, cyclosporin A and MMF were kindly supplied by Chong Kun Dang Pharmaceutical Corp.

MTT assay. The anti-cancer activity of the drugs on Huh7, HEP3B, SNU387 and SNU449 cells were determined using an MTT assay (Sigma-Aldrich; Merck KGaA; cat. no. M2003) to assess their cytotoxic effects, as previously described $(5,17)$. For each drug, two different doses were assessed; sirolimus (5 and $25 \mathrm{ng} / \mathrm{ml}$ ), tacrolimus (5 and $25 \mathrm{ng} / \mathrm{ml}$ ), cyclosporine A (100 and $500 \mathrm{ng} / \mathrm{ml}$ ) and MMF (500 and 1,000 ng). Each dose used was the clinically recommended trough level and clinically applicable maximum trough level $(9,18)$. Cell viability was calculated using the following formula: [Cell viability $(\%)=$ Mean optical density (OD)/Control OD x100\%]. The proliferative index was shown as a percentage relative to the control cells. All experiments were performed in triplicate.

Cell cycle arrest. Huh7 cells were seeded at a density of $1.5 \times 10^{5}$ into 6-well plates. After $24 \mathrm{~h}$, cells were treated with sirolimus ( 5 or $25 \mathrm{ng} / \mathrm{ml}$ ), tacrolimus ( 5 or $25 \mathrm{ng} / \mathrm{ml}$ ), MMF (500 or $1,000 \mathrm{ng} / \mathrm{ml}$ ) or cyclosporine A (100 or $500 \mathrm{ng} / \mathrm{ml}$ ). After 24 or $48 \mathrm{~h}$, cells were harvested by trypsinization, and digestion was stopped by adding fresh media. Cells were washed twice in cold PBS and fixed with $80 \%$ ethanol at $4^{\circ} \mathrm{C}$ for $1 \mathrm{~h}$. After washing with PBS, cells were stained with propidium iodide (PI; $50 \mathrm{mg} / \mathrm{ml}$ ) at room temperature for $5 \mathrm{~min}$ and treated with RNAse A $(20 \mathrm{mg} / \mathrm{ml})$. The DNA profile of cell populations was determined by flow cytometry. All experiments were repeated at least three times.

Western blotting. Western blot was performed as described previously (19). Total protein was extracted from cells using RIPA lysis buffer supplemented with $1 \mathrm{mg} / \mathrm{ml}$ aprotinin, $10 \mu \mathrm{l} / \mathrm{ml}$ leupeptin, $1 \mathrm{mM}$ sodium vanadate and $1 \mathrm{mM}$ phenylmethylsulfonyl fluoride. The cell lysates were centrifuged at $10,000 \times \mathrm{g}$ at $4^{\circ} \mathrm{C}$ for $30 \mathrm{~min}$ and $10 \mu \mathrm{g}$ of each cell lysate were quantified using a Bradford assay (Biosesang) and loaded per lane on SDS-gels $(7.5,10$ or 12\%). Samples were resolved using SDS-PAGE and transferred to PVDF membranes (EMD Millipore). The membranes were blocked in TBS containing $0.1 \%(\mathrm{v} / \mathrm{v})$ Tween-20 (TBST; Bio-Rad Laboratories, Inc.) and $5 \%(\mathrm{w} / \mathrm{v})$ skimmed milk for $1 \mathrm{~h}$ at room temperature. Subsequently, the membranes were sequentially incubated with the indicated primary and secondary antibodies diluted in TBST containing 2\% (w/v) BSA (Invitrogen; Thermo Fisher Scientific, Inc.). Anti-phospho-(p-)mTOR (Ser2448) antibodies (Cell Signaling Technology, Inc.; cat. no. 2971; 1:1,000) and anti-GAPDH (Cell Signaling Technology, Inc.; cat. no. $2118 ; 1: 1,000)$ were the primary antibodies used, Samples were incubated with the primary antibodies overnight at $4^{\circ} \mathrm{C}$. Subsequently, samples were incubated with an anti-rabbit IgG, horseradish peroxidase-linked antibody (Cell Signaling Technology, Inc.; cat. no. 7074; 1:5,000) was used for $2 \mathrm{~h}$ at $4^{\circ} \mathrm{C}$. GAPDH was used as the loading control. Western blots were repeated three times. Densitometry analysis was performed using ImageJ (National Institutes of Health).

Statistical analysis. Statistical analysis was performed using GraphPad Prism version 6 (GraphPad Software, Inc.). Data 

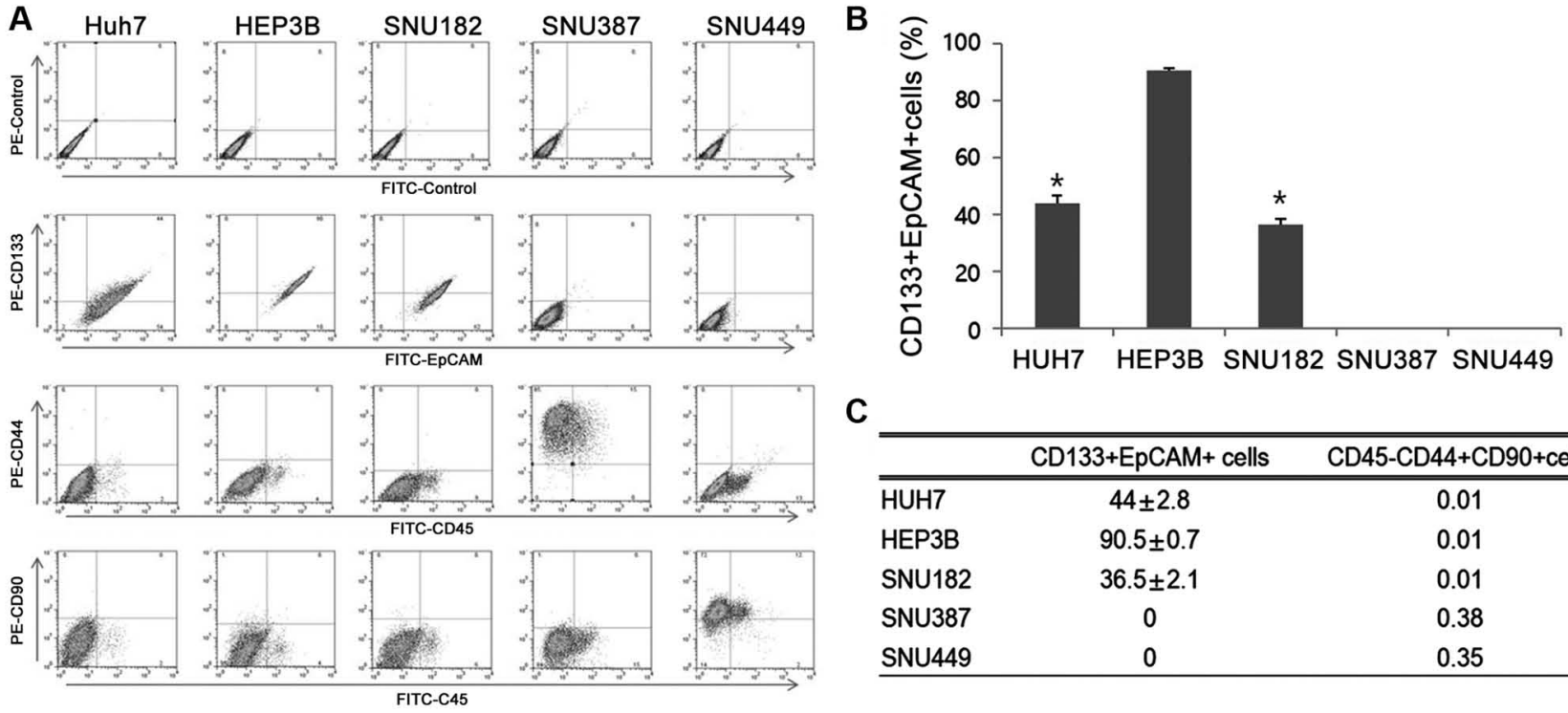

C

\begin{tabular}{lcc}
\hline \hline & CD133+EpCAM+ cells & CD45-CD44+CD90+cells \\
\hline HUH7 & $44 \pm 2.8$ & 0.01 \\
HEP3B & $90.5 \pm 0.7$ & 0.01 \\
SNU182 & $36.5 \pm 2.1$ & 0.01 \\
SNU387 & 0 & 0.38 \\
SNU449 & 0 & 0.35 \\
\hline
\end{tabular}

Figure 1. Differential expression of CSC markers in various hepatocellular carcinoma cell lines. (A) Representative dot plots showing the proportion of stained cells in each condition. (B) Bar graph showing the mean percentage of CD133+EpCAM+ cells in each cell line. (C) Expression of the well-established CSC markers, CD133 and EpCAM was notably high in Huh7, SNU182 and HEP3B cells. "P<0.05 vs. HEP3B. SNUH387 and SNUH449 cell lines did not express CD133 or EpCAM. CD44+CD90+ double positive CSCs, which are known to exhibit high degrees of proliferation, only accounted for $<0.5 \%$ in all cell lines. CSC, cancer stem cell; PE, phycoerythrin; FITC, fluorescein isothiocyanate; EpCAM, epithelial cell adhesion molecule.

are presented as the mean \pm the standard error off the mean. Differences between groups were compared using an ANOVA with a post-hoc and Tukey's honest significant difference test.

\section{Results}

Selection of CSC markers and cell lines in the various HCC cell lines. First, expression of four representative CSC markers (CD133, EpCAM, CD44 and CD90) in four HCC cell lines were assessed (Fig. 1A). The expression levels of CD133+EpCAM+ were notably higher in Huh7, HEP3B and SNU182 cells $(44 \pm 2.8,90.5 \pm 0.7$ and $36.5 \pm 2.1 \%$, respectively) compared with SNU387 and SNU449. SNUH387 and SNUH449 cell lines did not express either of these markers (Fig. 1B and C). Thus, SNUH387 and SNUH449 were not used for subsequent experiments. CD44+CD90+ double positive cells exhibit high degrees of proliferation in CSCs (20), but the proportion of cells attributed to this population was $<0.5 \%$ in all four cell lines (Fig. 1C). Due to the higher expression levels of CD133+EpCAM+ observed in the HEP3B cell line and the medium expression levels observed in the Huh7 cells, these cell lines were chosen for use in subsequent experiments. Huh7 and SNU182 cell lines showed roughly the same level of CD133+EpCAM+ expression, thus only the Huh7 cells were used for subsequent experiments (Fig. 1B and C).

Effect of various immune suppressants on proliferation and percentage of CSCs in HCC cell lines. Huh7 and HEP3B cell lines were used for the cell viability assays in order to determine the effects of different immunosuppressants including sirolimus, tacrolimus, cyclosporine A and MMF on CSCs. For each drug, two different doses were assessed; sirolimus (5 and $25 \mathrm{ng} / \mathrm{ml}$ ), tacrolimus (5 and $25 \mathrm{ng} / \mathrm{ml}$ ), cyclosporine A (100 and $500 \mathrm{ng} / \mathrm{ml}$ ) and MMF (500 and 1,000 ng). The control cells were treated with saline without immunosuppressants for each cell line. An MTT assay was used to measure cell proliferation and survival. The proliferation index was calculated by comparing the expression of the treated cells with the respective control treated cells.

The proliferation rate of Huh7 cells was significantly reduced by sirolimus $(5 \mathrm{ng} / \mathrm{ml}, 50.70 \pm 1.86 ; 25 \mathrm{ng} / \mathrm{ml}, 47.50 \pm 0.96$ ) and MMF (500 ng, 72.57 $\pm 2.13 ; 1,000 \mathrm{ng}, 58.88 \pm 2.54$ ) when compared with the control cells. However, neither cell line was affected by tacrolimus or cyclosporine A. The Huh7 cell line was considerably more sensitive than HEP3B cells to sirolimus. The proliferation rate of HEP3B was also decreased by sirolimus $(5 \mathrm{ng} / \mathrm{ml}, 87.62 \pm 3.96 ; 25 \mathrm{ng} / \mathrm{ml}, 87.65 \pm 5.04)$ and MMF (500 ng, 83.93 $\pm 10.40 ; 1,000$ ng, 68.74 \pm 5.84 ) but tacrolimus and cyclosporine $\mathrm{A}$ did not affect the proliferation rate of HEP3B cells (Fig. 2A).

To determine any changes to the proportion of CSCs following treatment with immunosuppressants in Huh7 and HEP3B cells, the expression levels of CD133+EpCAM+ in each cell type following treatment with immunosuppressants was measured. The percentage of CD133+EpCAM+ expressing cells was significantly increased in Huh7 cells when treated with either sirolimus $(5 \mathrm{ng} / \mathrm{ml}, 192.86 \pm 23.57 \% ; 25 \mathrm{ng} / \mathrm{ml}$, $205.36 \pm 3.69 \%$ ) and MMF (500 ng, 191.07 $\pm 11.90 \% ; 1,000 \mathrm{ng}$, $201.79 \pm 6.25 \%)$ treatment compared with the respective control cells. However, the proliferation of CD133+EpCAM+ expressing cells was significantly decreased by both sirolimus and MMF. In the HEP3B cell line the percentage of CD133+EpCAM+ cells was not significantly altered by sirolimus (5 ng/ml, 107.81 $\pm 12.30 \% ; 25 \mathrm{ng} / \mathrm{ml}, 104.69 \pm 14.78 \%$ ), but proliferation was increased by MMF (500 ng, 129.69 $\pm 5.11 \%$; $1,000 \mathrm{ng}, 131.25 \pm 3.40 \%$ ) (Fig. 2B).

G1 or S phase arrest in Huh7 and HEP3B cells treated with sirolimus or MMF. As shown in Fig. 2, sirolimus and MMF both reduced the proliferation rate of Huh7 cells. To determine 

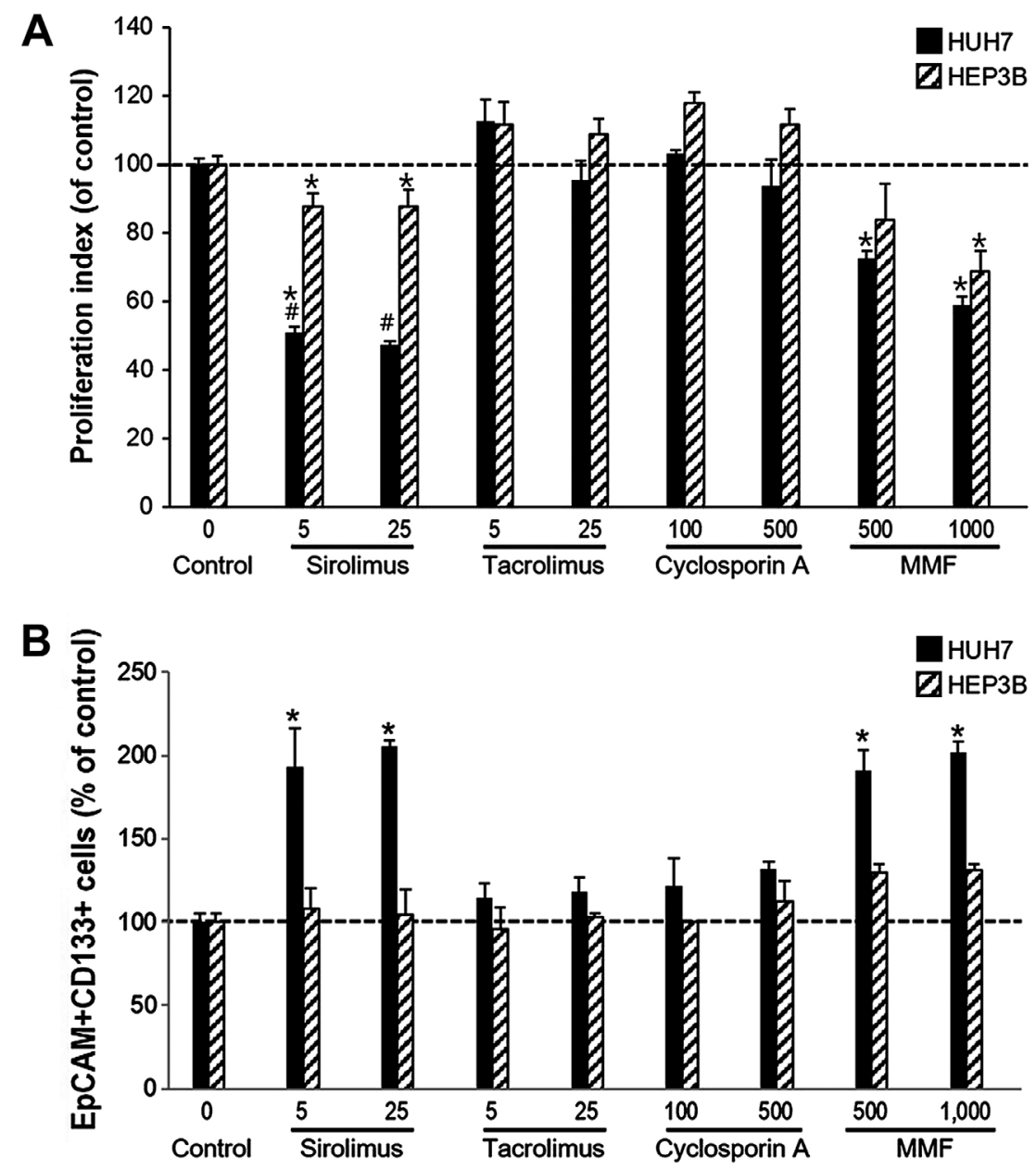

Figure 2. Effect of immunosuppressants on cell proliferation. (A) Proliferation index of Huh7 was significantly decreased by sirolimus and MMF compared with the respective control cells, but was not affected by tacrolimus and cyclosporin A. The Huh7 cell line was notably more sensitive than HEP3B to sirolimus and MMF. Proliferation of HEP3B was also decreased by sirolimus and MMF. However, tacrolimus and cyclosporin A did not have a significant effect on proliferation of the HEP3B cells. (B) Percentage of CD133+EpCAM+ expressing cells was significantly increased in Huh7 cells treated with sirolimus and MMF. In HEP3B cells, the percentage of CD133+EpCAM+ cells was not affected by sirolimus, and significantly increased by MMF at both concentrations. ${ }^{*} \mathrm{P}<0.05$ vs. respective control; $\mathrm{P}<0.05$ vs. HEP3B treated at the same respective concentration. MMF, mycophenolate mofetil.

the mechanism of inhibition of growth mediated by sirolimus and MMF treatment, cell cycle analyses were performed in both Huh7 and HEP3B cells using PI staining. Sirolimus and MMF are both known to induce cell cycle arrest, thus, their effects on CD133+EpCAM+ or CD133-EpCAM- populations from Huh7 cells were analyzed. The degree of cell cycle arrest of the total Huh7 cell population or CD133+EpCAM+ or CD133-EpCAM- populations were determined by gating for double positive or double negative events following treatment with immunosuppressants. Cell cycle arrest in HEP3B cells was not measured as $>90 \%$ of the population were CD133+EpCAM+ cells.

In $\mathrm{HuH} 7$ cells, sirolimus increased G0-G1 arrest (5 ng/ml, 63.44\%; $25 \mathrm{ng} / \mathrm{ml}, 71.92 \%$; control, 52.81\%) and the proportion of the CD133-EpCAM- population $(5 \mathrm{ng} / \mathrm{ml}$, 77.94\%; $25 \mathrm{ng} / \mathrm{ml}, 84.01 \%$; control, 68.48\%). However, the CD133+EpCAM+ population only exhibited G1 arrest at the higher doses of sirolimus $(5 \mathrm{ng} / \mathrm{ml}, 46.63 \% ; 25 \mathrm{ng} / \mathrm{ml}, 61.62 \%$; control, 44.62\%). In contrast, arrest at the S-phase was induced by both doses of MMF in Huh7 cells in the CD133-EpCAMand CD133+EpCAM+ populations. Cell cycle arrest was not induced by tacrolimus or cyclosporine A treatment (data not shown). These results shows that the inhibitory mechanism of sirolimus or MMF in Huh7 is mediated by cell cycle arrest at the G1 or S phase (Fig. 3).

mTOR pathway is regulated by immunosuppressants. mTOR has been shown to be a key molecule involved in the PTEN/PI3K/mTOR signaling pathway and serves a critical role in controlling cell proliferation and survival. The protein expression levels of mTOR and p-mTOR in Huh7 and HEP3B cells were measured following treatment with various immunosuppressants. In both Huh7 and HEP3B cells, the protein expression levels of mTOR were significantly decreased by treatment with sirolimus $(0.42 \pm 0.11$ and $0.49 \pm 0.17$, respectively) when compared with the respective control treated cell lines. However, the protein expression levels of mTOR were not affected notably by treatment with tacrolimus, cyclosporin A and MMF. In HEP3B cells, the protein expression levels of mTOR were reduced by sirolimus $(0.44 \pm 0.08)$, MMF $(0.79 \pm 0.14)$ and cyclosporin A $(0.73 \pm 0.002$; Fig. $4 \mathrm{~A}$ and B). However, tacrolimus did not affect the expression levels of mTOR. These results are summarized in Table I. 
A
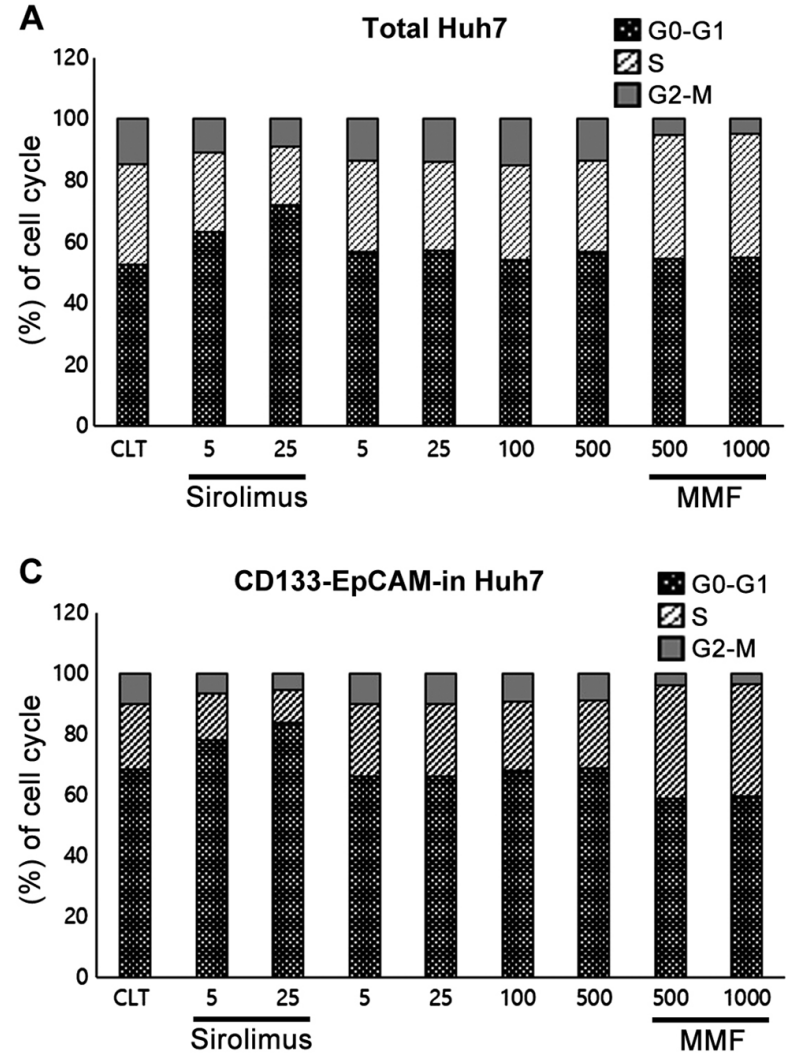

B

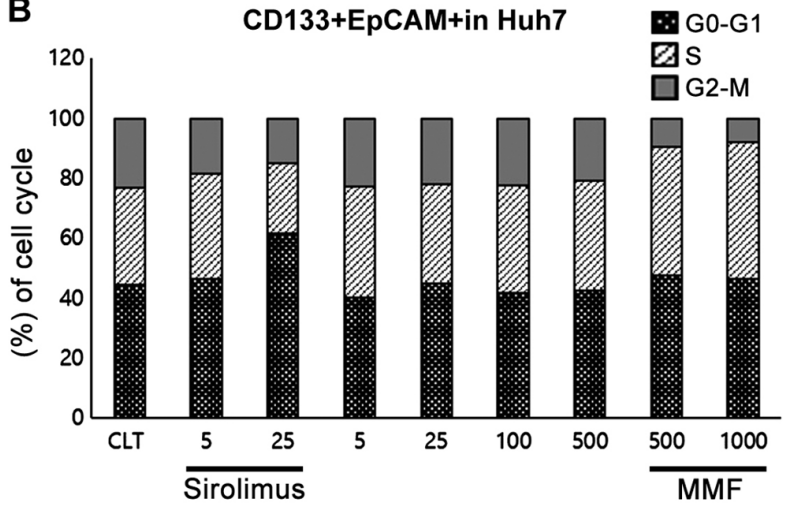

D

\begin{tabular}{c|c|c|c}
\hline \hline \multirow{2}{*}{ \% G0-G1 arrest } & \multirow{2}{*}{ CTL } & \multicolumn{2}{|c}{ Sirolimus } \\
\cline { 3 - 4 } & & $5 \mathrm{ng}$ & $25 \mathrm{ng}$ \\
\hline \hline CD133+EpCAM+/ Huh7 & 44.62 & 46.63 & 61.62 \\
CD133-EpCAM- / Huh7 & 68.48 & 77.94 & 84.01 \\
Total Huh7 & 52.81 & 63.44 & 71.92 \\
\hline \hline & \multirow{2}{|c}{ MMF } \\
\hline \multirow{2}{*}{ \% S arrest } & CTL & $500 \mathrm{ng}$ & $1,000 \mathrm{ng}$ \\
\cline { 3 - 4 } & & 42.65 & 45.55 \\
\hline CD133+EpCAM+/ Huh7 & 32.28 & 37.51 & 36.98 \\
CD133-EpCAM- / Huh7 & 21.64 & 40.31 & 40.17 \\
Total Huh7 & 32.48 & \multicolumn{2}{|c}{}
\end{tabular}

Figure 3. G1 or S phase cell-cycle arrest in Huh7 cells treated with sirolimus or MMF. (A) In HuH7 cells, sirolimus increased G0-G1 arrest (5 ng/ml, $63.44 \%$; $25 \mathrm{ng} / \mathrm{ml}, 71.92 \%$; control, 52.81\%). (B) G1 arrest was only observed in the CD133+EpCAM+ population at higher doses of sirolimus (5 ng/ml, $46.63 \%$; $25 \mathrm{ng} / \mathrm{ml}, 61.62 \%$; control, 44.62\%). (C) Sirolimus increased G0-G1 arrest in the CD133-EpCAM- population of HuH7 cells $(5 \mathrm{ng} / \mathrm{ml}, 77.94 \%$; $25 \mathrm{ng} / \mathrm{ml}$, 84.01\%; control, 68.48\%). (A-D) In contrast, S phase arrest by MMF was induced at all doses in the total Huh7, CD133-EpCAM- and CD133+EpCAM+ populations, and no cell cycle arrest was observed in the tacrolimus or cyclosporine A treated cells. These results demonstrated that the inhibitory mechanism of sirolimus or MMF on Huh7 proliferation was associated with cell cycle arrest at the G1 and/or S phase. MMF, mycophenolate mofetil; EpCAM, epithelial cell adhesion molecule.

A

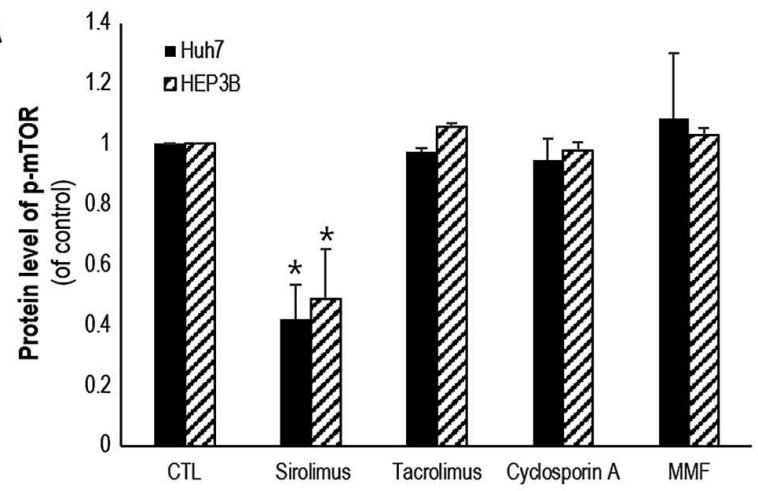

B

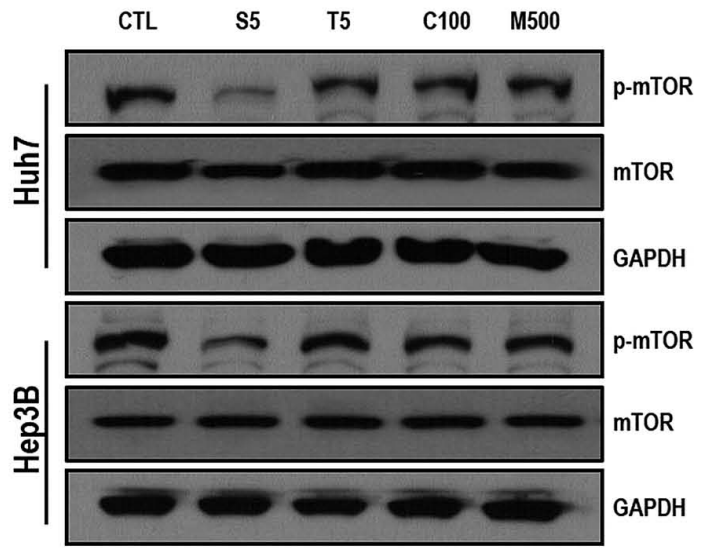

Figure 4. mTOR signaling is regulated by sirolimus. (A and B) Densitometry analysis and representative blots of the protein expression levels of p-mTOR in Huh7 and Hep3B cells were significantly decreased by sirolimus compared with control cells but the protein expression levels of mTOR was not affected by tacrolimus, cyclosporin A or MMF. "P<0.05 vs. respective control. mTOR, mammalian target of rapamycin; p-, phospho; MMF, mycophenolate mofetil.

\section{Discussion}

Tumor recurrence following LT is the leading cause of death in patients with HCC, and the incidence of recurrence is $15-24 \%(4,5)$. Additionally, tumor progression may be more rapid and aggressive in patients administered immunosuppressants when they have received a LT (6). Therefore, the role of immunosuppressive therapy in HCC recurrence remains a challenging issue, as a balance between graft survival and HCC recurrence has to be taken into consideration (5).

CNIs dose-dependently increase the risk of HCC recurrence, despite the fact that these are the primary immunosuppressive agents administered to LT recipients $(21,22)$. Although several centers use mTORIs in patients with 
Table I. Summary of effects of MMF and sirolimus on progression of hepatocellular cell lines.

Huh7

HEP3B

\begin{tabular}{|c|c|c|c|c|}
\hline \multirow[b]{2}{*}{ Cellular process } & & \\
\hline & Sirolimus & MMF & Sirolimus & MMF \\
\hline \multicolumn{5}{|l|}{ Proliferation } \\
\hline Overall & Inhibition & Inhibition & Inhibition & Inhibition \\
\hline CD133+/EpCAM+ population & Increased & Increased & No change & Slightly increased \\
\hline \multicolumn{5}{|l|}{ Cell cycle arrest } \\
\hline Overall & G1 arrest & $\mathrm{S}$ arrest & ND & ND \\
\hline CD133+/EpCAM+ & Minimal G1 arrest at highest dose & $\mathrm{S}$ arrest & ND & ND \\
\hline CD133-/EpCAM- & G1 arrest & $\mathrm{S}$ arrest & ND & ND \\
\hline p-mTOR protein expression & Reduction & No change & Reduction & No change \\
\hline
\end{tabular}

MMF, mycophenolate mofetil; EpCAM, epithelial cell adhesion molecule; ND, not detected.

advanced HCC, there is a lack of prospective, randomized control trials to support any recommendation for their use for this purpose. At the same time, as the patient will require life-long use of immunosuppressants following transplantation, careful selection of appropriate drugs is required. It is therefore important to determine which treatment regimens provide the optimal combination of both immunosuppressive and tumor-suppressive effects.

Whether mTORIs can reduce HCC recurrence following LT is still controversial. Even though some retrospective and prospective studies have reported the positive results of mTORIs in combination with a CNI $(10,11,23,24)$, it is still unclear whether this benefit is from the direct effect of mTORIs or the indirect effect of reducing CNI doses. Furthermore, one prospective randomized direct comparative study (SiLVER study) of a rapamycin inhibitor demonstrated negative outcomes (25). The present study provides a theoretical background with regard to this controversial issue. Based on the results of the present study, mTORIs can reduce the rate of recurrence in patients with HCC through reducing cell proliferation; however, it cannot reduce the absolute rate of recurrence, as it does not inhibit the actions of CSCs (CD133+EpCAM+ cells). To prevent recurrence following LT, the targeted control of the remnant or circulating CSCs should be considered in balance with the appropriate immunosuppressant to protect the graft.

Several CSC biomarkers (for example, CD133, EpCAM, CD90, CD24 and Nanog) have been identified in HCC $(26,27)$. CD133, was originally classified as a hematopoietic stem cell marker and CD133 has also been used to isolate stem-like cells from HCC cell lines $(25,26)$. Interestingly, analysis using flow cytometry has shown that the percentage of CD133+ cells differs significantly amongst several HCC cell lines (from 1 to $>90 \%$ ) (28). EpCAM is also considered a CSC marker (29) and $>35 \%$ of HCC tissues exhibit positive EpCAM expression (30-32). Luo et al (33) showed that CD90+ cells not only possess a high affinity to form tumors, but also other features of CSCs, such as extensive proliferation, differentiation, chemo-resistance, and invasive and metastatic capacity. As such CD133, EpCAM and CD90 are ideal candidates for investigation as CSC markers in HCC cell lines. Therefore, in the present study the expression of these markers were used to determine CSCs in the HCC cell lines.

The aim of the present study was to identify an improved immunosuppression regimen for LT patients with $\mathrm{HCC}$ in vitro using several $\mathrm{HCC}$ cell lines and their respective CSC populations. Huh7 and HEP3B cell lines were used as they were shown to possess high levels of CD133+EpCAM+ cell populations, which were considered CSCs. Interestingly, sirolimus and MMF effectively reduced the proliferation of Huh7 and HEP3B cells, but the percentage of CD133+EpCAM+ expressing cells were significantly increased in Huh7 following treatment in both cell lines. This result suggested that sirolimus and MMF did not have an inhibitory effect on the CD133+EpCAM+ subpopulation of cells in the Huh7 cell line. The percentage of CD133+EpCAM+ expressing cells was not significantly increased following treatment with sirolimus, but was significantly increased by MMF in HEP3B cells. Over $90 \%$ of HEP3B cells were shown to be CD133+EpCAM+; as the majority of the HEP3B cells were $\mathrm{CD} 133+\mathrm{EpCAM}+$, the proportion of CD133+EpCAM+ cells in HEP3B was unlikely to be notably affected by sirolimus or MMF. However, these results do show that sirolimus and MMF reduced the proliferation of cancer cells, and by doing so may contribute to increasing survival times in LT patients with HCC. However, sirolimus and MMF may not be sufficient to reduce the recurrence rate, as there remain CSCs following LT. As such even with the use of sirolimus or MMF, which exhibit anti-proliferative effects in HHC, these drugs may not be able to suppress the properties of the CSC population.

The results from Yang et al (34) were in agreement with the results of the present study. Luo et al (33) demonstrated that rapamycin significantly increased both the proportion of CD133+ cells in vitro and in vivo, but also increased the expression of stem cell-like genes $(34,35)$. However, there are no studies showing the effects of MMF on the levels of CD133 cell populations in cancer cells. As such, the present study is the first to provide this data. The results of the present study suggest that the inhibitory mechanism of sirolimus and MMF on Huh7 and HEP3B cells were largely associated with cell cycle arrest at the $\mathrm{G} 1$ or $\mathrm{S}$ phase. It was also confirmed that the protein expression levels of mTOR, a key molecule in the 
PTEN/PI3K/mTOR signaling pathway, which serves a critical role in controlling cell proliferation and survival, was significantly decreased following sirolimus treatment in Huh7 and HEP3B cells compared with the control cells.

Higher doses of mTORIs may provide an enhanced anticancer effect; however, there are no clinical studies investigating the minimal effective concentration for use following LT for HCC to provide maximal anti-tumor effect. Furthermore, the trough level of most clinical studies using mTORIs was 3-8 $\mathrm{ng} / \mathrm{ml}$, as the adverse events associated with mTORIs appear to be dose-related (35). Therefore, the 3-8 $\mathrm{ng} / \mathrm{ml}$ range has been demonstrated to offer the optimal risk-benefit profile, even in patients with HCC. In summary, the present study showed that HCC cells express different levels of CSC markers, such as CD133 or EpCAM, and they have different sensitivities to immunosuppressants. Sirolimus effectively reduced the proliferation of various cancer cell lines, but failed to affect the proportion of the CD133+EpCAM+ cells specifically. Therefore, these immunosuppressant agents should be further assessed in vivo for potential use in patients, to help regulate CSC populations, and thus reduce the risk of recurrence $\mathrm{HCC}$.

\section{Acknowledgements}

We deeply appreciate the contributions from Ms Kwang-Sook Shin and Ms Yang-Hee Kim (Department of Surgery, Seoul National University College of Medicine) for their assistance with the molecular work performed in the present study.

\section{Funding}

This study was supported by funding from the Seoul National University Hospital research fund by (grant no. 0320110450) and Basic Science Research Program through the National Research Foundation of Korea funded by the Ministry of Science, ICT and Future Planning (grant no. NRF-2016R1A2B4010665).

\section{Availability of data and materials}

The datasets used and/or analyzed during the present study are available from the corresponding author on reasonable request.

\section{Authors' contributions}

HK, KWL and KSS designed the study, performed the experiments, analyzed the data and wrote the manuscript. SCO, MYP, SS and XLJ performed the experiments. SKH and KCY analyzed the data. NJY designed the study and analyzed the data. All authors read and approved the final manuscript.

\section{Ethics approval and consent to participate}

Not applicable.

\section{Patient consent for publication}

Not applicable.

\section{Competing interests}

The authors declare that they have no competing interests.

\section{References}

1. Torre LA, Bray F, Siegel RL, Ferlay J, Lortet-Tieulent J and Jemal A: Global cancer statistics, 2012. CA Cancer J Clin 65: 87-108, 2015

2. Parkin DM, Bray F, Ferlay $J$ and Pisani P: Global cancer statistics, 2002. CA Cancer J Clin 55: 74-108, 2005.

3. Park MS, Lee KW, Yi NJ, Choi YR, Kim H, Hong G, Suh KS, Kwon CH, Joh JW and Lee SK: Optimal tailored screening protocol after living donor liver transplantation for hepatocellular carcinoma. J Korean Med Sci 29: 1360-1366, 2014.

4. Mukherjee S and Mukherjee U: A comprehensive review of immunosuppression used for liver transplantation. J Transplant 2009: 701464, 2009.

5. Moini M, Schilsky ML and Tichy EM: Review on immunosuppression in liver transplantation. World J Hepatol 7: 1355-1368, 2015.

6. Liu Z, Chen Y, Tao R, Xv J, Meng J and Yong X: Tacrolimus-based versus cyclosporine-based immunosuppression in hepatitis $\mathrm{C}$ virus-infected patients after liver transplantation: A meta-analysis and systematic review. PLoS One 9: e107057, 2014.

7. Rodríguez-Perálvarez $\mathrm{M}$, De la Mata $\mathrm{M}$ and Burroughs $\mathrm{AK}$ : Liver transplantation: Immunosuppression and oncology. Curr Opin Organ Transplant 19: 253-260, 2014.

8. Chen K, Man K, Metselaar HJ, Janssen HL, Peppelenbosch MP and Pan Q: Rationale of personalized immunosuppressive medication for hepatocellular carcinoma patients after liver transplantation. Liver Transpl 20: 261-269, 2014.

9. Lee KW, Seo YD, Oh SC, Suh SW, Jeong J, Kim H, Yi NJ and Suh KS: What is the best immunosuppressant combination in terms of antitumor effect in hepatocellular carcinoma? Hepatol Res 46: 593-600, 2016.

10. Menon KV, Hakeem AR and Heaton ND: Meta-analysis: Recurrence and survival following the use of sirolimus in liver transplantation for hepatocellular carcinoma. Aliment Pharm Ther 37: 411-419, 2013.

11. Jeng LB, Thorat A, Hsieh YW, Yang HR, Yeh CC, Chen TH, Hsu SC and $\mathrm{Hsu} \mathrm{CH}$ : Experience of using everolimus in the early stage of living donor liver transplantation. Transplant Proc 46: 744-748, 2014.

12. Finn RS: Current and future treatment strategies for patients with advanced hepatocellular carcinoma: Role of mTOR inhibition. Liver Cancer 1: 247-256, 2012.

13. Yin S, Li J, Hu C, Chen X, Yao M, Yan M, Jiang G, Ge C, Xie H, Wan D, et al: CD133 positive hepatocellular carcinoma cells possess high capacity for tumorigenicity. Int J Cancer 120: 1444-1450, 2007.

14. Suetsugu A, Osawa Y, Nagaki M, Moriwaki H, Saji S, Bouvet M and Hoffman RM: Simultaneous color-coded imaging to distinguish cancer 'stem-like' and non-stem cells in the same tumor. J Cell Biochem 111: 1035-1041, 2010.

15. Ma S, Chan KW, Hu L, Lee TK, Wo JY, Ng IO, Zheng BJ and Guan XY: Identification and characterization of tumorigenic liver cancer stem/progenitor cells. Gastroenterology 132: 2542-2556, 2007.

16. Reya T, Morrison SJ, Clarke MF and Weissman IL: Stem cells, cancer, and cancer stem cells. Nature 414: 105-111, 2001.

17. Kumar P, Nagarajan A and Uchil PD: Analysis of cell viability by the MTT assay. Cold Spring Harb Protoc 2018, 2018.

18. Mussin N, Oh SC, Lee KW, Park MY, Seo S, Yi NJ, Kim H, Yoon KC, Ahn SW, Kim HS, et al: Sirolimus and metformin synergistically inhibits colon cancer in vitro and in vivo. J Korean Med Sci 32: 1385-1395, 2017.

19. Hui IC, Tung EK, Sze KM, Ching YP and Ng IO: Rapamycin and CCI-779 inhibit the mammalian target of rapamycin signalling in hepatocellular carcinoma. Liver International 30: 65-75, 2010.

20. Ji J and Wang XW: Clinical implications of cancer stem cell biology in hepatocellular carcinoma. Semin Oncol 39: 461-472, 2012 .

21. Sgourakis G and Dedemadi G: Corticosteroid-free immunosuppression in liver transplantation: An evidence-based review. World J Gastroenterol 20: 10703-10714, 2014.

22. Turner AP and Knechtle SJ: Induction immunosuppression in liver transplantation: A review. Transpl Int 26: 673-683, 2013. 
23. Vivarelli M, Dazzi A, Cucchetti A, Gasbarrini A, Zanello M Di Gioia P, Bianchi G, Tamè MR, Gaudio MD, Ravaioli M, et al: Sirolimus in liver transplant recipients: A large single-center experience. Transplant Proc 42: 2579-2584, 2010.

24. Thorat A, Jeng LB, Yang HR, Yeh CC, Hsu SC, Chen TH and Poon KS: Assessing the role of everolimus in reducing hepatocellular carcinoma recurrence after living donor liver transplantation for patients within the UCSF criteria: Re-inventing the role of mammalian target of rapamycin inhibitors. Ann Hepatobiliary Pancreat Surg 21: 205-211, 2017.

25. Geissler EK, Schnitzbauer AA, Schlitt HJ and Si LSG: Lack of benefits of mammalian target of rapamycin inhibitor in patients transplanted for hepatocellular carcinoma: Is this the end of the story? Liver Transpl 22: 1162-1163, 2016.

26. Yamashita T, Ji J, Budhu A, Forgues M, Yang W, Wang HY, Jia H, Ye Q, Qin LX, Wauthier E, et al: EpCAM-positive hepatocellular carcinoma cells are tumor-initiating cells with stem/progenitor cell features. Gastroenterology 136: 1012-1024, 2009.

27. Na DC, Lee JE, Yoo JE, Oh BK, Choi GH and Park YN: Invasion and EMT-associated genes are up-regulated in B viral hepatocellular carcinoma with high expression of CD133-human and cell culture study. Exp Mol Pathol 90: 66-73, 2011.

28. Zhu Z, Hao X, Yan M, Yao M, Ge C, Gu J and Li J: Cancer stem/progenitor cells are highly enriched in CD133+CD44+ population in hepatocellular carcinoma. Int J Cancer 126: 2067-2078, 2010.
29. Winter MJ, Nagelkerken B, Mertens AE, Rees-Bakker HA, Briaire-de Bruijn IH and Litvinov SV: Expression of Ep-CAM shifts the state of cadherin-mediated adhesions from strong to weak. Exp Cell Res 285: 50-58, 2003.

30. Kim JW, Ye QH, Forgues M, Chen YD, Budhu A, Sime J, Hofseth LJ, Kaul R and Wang XW: Cancer-associated molecular signature in the tissue samples of patients with cirrhosis. Hepatology 39: 518-527, 2004.

31. de Boer CJ, Van Krieken JH, Janssen-Van Rhijn CM and Litvinov SV: Expression of Ep-CAM in normal, regenerating, metaplastic, and neoplastic liver. J Pathol 188: 201-206, 1999.

32. Ruck P, Wichert G, Handgretinger R and Kaiserling E: Ep-CAM in malignant liver tumours. Journal of Pathology 191: 102-103, 2000.

33. Luo J, Wang P, Wang R, Wang J, Liu M, Xiong S, Li Y and Cheng B: The Notch pathway promotes the cancer stem cell characteristics of CD90+ cells in hepatocellular carcinoma. Oncotarget 7: 9525-9537, 2016.

34. Yang Z, Zhang L, Ma A, Liu L, Li J, Gu J and Liu Y: Transient mTOR inhibition facilitates continuous growth of liver tumors by modulating the maintenance of CD133+ cell populations. PLoS One 6: e28405, 2011.

35. Matsumoto K, Arao T, Tanaka K, Kaneda H, Kudo K, Fujita Y, Tamura D, Aomatsu K, Tamura T, Yamada Y, et al: mTOR signal and hypoxia-inducible factor-1 alpha regulate CD133 expression in cancer cells. Cancer Res 69: 7160-7164, 2009. 